\title{
Adding Bismuth to Rabeprazole-Based First-Line Triple Therapy Does Not Improve the Eradication of Helicobacter pylori
}

\author{
Meng-Chieh Wu, ${ }^{1,2}$ Yao-Kuang Wang, ${ }^{1}$ Chung-Jung Liu, ${ }^{1}$ Fang-Jung Yu, ${ }^{1,3}$ Fu-Chen Kuo, \\ Min-Li Liu, ${ }^{5}$ Chao-Hung Kuo, ${ }^{1,3}$ Deng-Chyang Wu, ${ }^{1,2,3}$ Yao-Kang Huang, ${ }^{6}$ and I-Chen Wu ${ }^{1,3}$ \\ ${ }^{1}$ Division of Gastroenterology, Department of Internal Medicine, Kaohsiung Medical University Hospital, Kaohsiung, Taiwan \\ ${ }^{2}$ Department of Internal Medicine, Kaohsiung Municipal Ta-Tung Hospital, Kaohsiung, Taiwan \\ ${ }^{3}$ Faculty of Medicine, Department of Medicine, College of Medicine, Kaohsiung Medical University, Kaohsiung, Taiwan \\ ${ }^{4}$ School of Medicine, College of Medicine, E-Da Hospital, I-Shou University, Kaohsiung, Taiwan \\ ${ }^{5}$ Department of Pharmacy, E-Da Cancer Hospital, Kaohsiung, Taiwan \\ ${ }^{6}$ Division of Gastroenterology, Department of Internal Medicine, Ten-Chan General Hospital, Chung-Li, Taoyuan, Taiwan
}

Correspondence should be addressed to Yao-Kang Huang; paulyhwong1997@gmail.com and I-Chen Wu; minicawu@gmail.com

Received 15 March 2017; Accepted 11 June 2017; Published 16 July 2017

Academic Editor: Francesco Selvaggi

Copyright (c) 2017 Meng-Chieh Wu et al. This is an open access article distributed under the Creative Commons Attribution License, which permits unrestricted use, distribution, and reproduction in any medium, provided the original work is properly cited.

\begin{abstract}
This randomized controlled study aimed to evaluate whether adding bismuth to the standard first-line triple therapy could improve the eradication rate of Helicobacter pylori. A total of 162 patients with Helicobacter pylori infection were randomly assigned to either the 7-day triple therapy group (RAK regimen: rabeprazole $20 \mathrm{mg}$, amoxicillin $1 \mathrm{~g}$, and clarithromycin $500 \mathrm{mg}$ bid; $n=81)$ or the bismuth plus triple therapy group $(n=81)$. In the RBAK group, bismuth subcitrate $360 \mathrm{mg}$ twice daily was added to the RAK regimen. A follow-up endoscopy or urea breath test was performed at least 4 weeks after eradication to confirm the treatment efficacy. Comparable compliance and Helicobacter pylori eradication rates were observed in both groups in either intention-to-treat [RAK 72.8\% (59/81) versus RBAK 77.8\% $(63 / 81) ; p=0.47$ ] or per protocol analysis [RAK 74.7\% (59/79) versus RBAK 81.8\% (63/77); $p=0.26$ ]. Adverse effects were commonly reported (50.6\% for both groups) although most of these did not cause cessation of treatment. The resistance rate was $27.2 \%$ for metronidazole and $12.3 \%$ for clarithromycin. Adding bismuth to the standard 7-day triple therapy did not substantially increase the eradication rate. Further study is needed clarifying whether extending the duration of RBAK regimen to 10-14 days can lead to a better result.
\end{abstract}

\section{Introduction}

Helicobacter pylori (H. pylori) is an important cause of gastritis, peptic ulcer, and gastric cancer [1]. In 1997, the Maastricht Consensus Meeting suggested standard triple therapy including proton pump inhibitor (PPI), clarithromycin, and amoxicillin given twice daily for 7 days as the first-line treatment for $H$. pylori with treatment regimens achieving an eradication rate of over $80 \%$ [2]. However, with increasing antibiotic resistance and decreasing efficacy of the standard triple therapy globally, identification of a better first-line regimen has become an essential issue. A higher clarithromycin resistance rate results in lower treatment efficacy, which falls below $85 \%$ for standard 7-day triple therapy if the prevalence of clarithromycin resistance is 15\% [3]. In 2010, Graham and colleagues indicated that the eradication rate of triple therapy was $>80 \%$ in only $40 \%$ of studies reviewed and $>85 \%$ in $18 \%$ of such studies [4]. Maastricht V Consensus also suggested avoiding standard triple therapy as first-line regimen in areas of high clarithromycin resistance $(>15 \%)$ [5].

Bismuth-containing therapy is one of the proposed substitutes to replace standard triple therapy [5]. Bismuth exerts its antimicrobial effect by suppressing $H$. pylori, although it is unable to eliminate the organism [6]. In addition to clarithromycin, there is an increasing resistance to metronidazole and levofloxacin in many countries [7]. In Taiwan, 


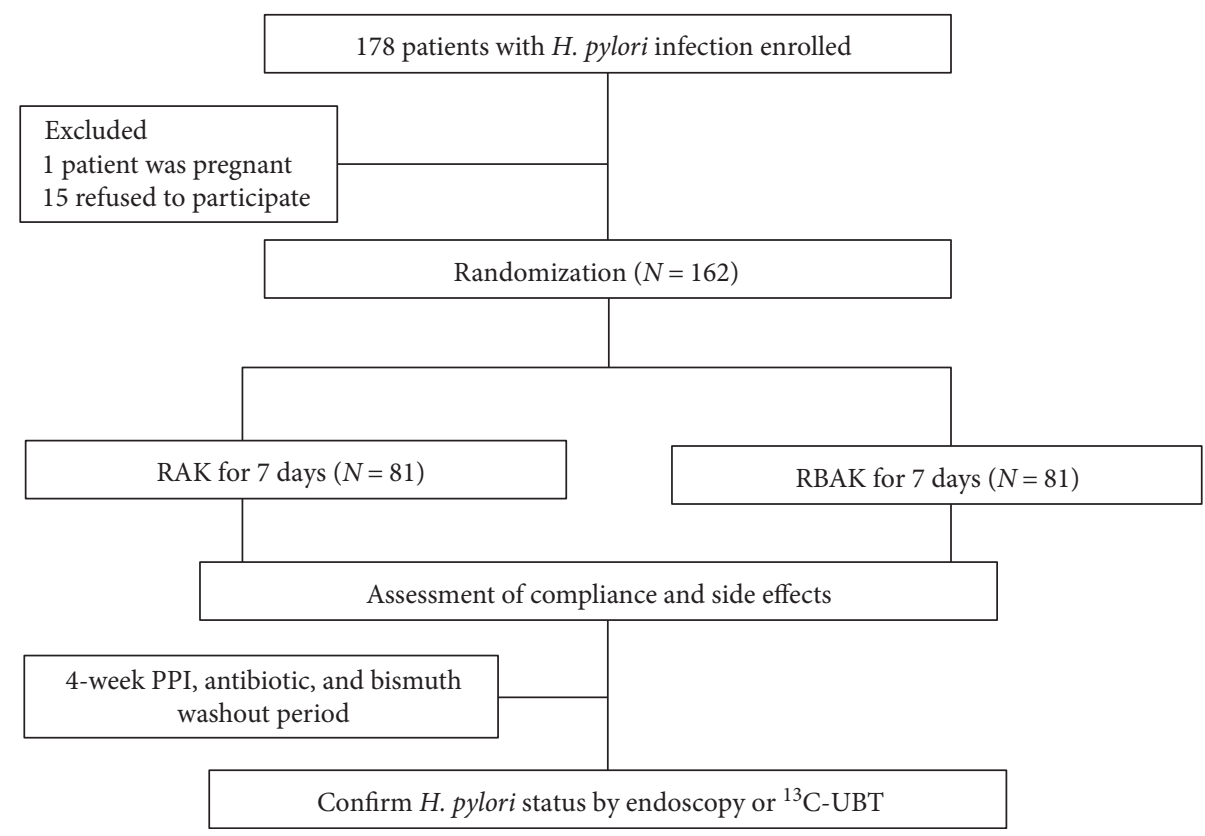

Figure 1: Flowchart.

the clarithromycin resistance rate increased from $10.1 \%$ in 2000-2007 to $12.8 \%$ in 2011-2012 [8]. Recent studies reported variable eradication rates $(77.3-81.6 \%$ in intention-to-treat [ITT] analysis) using standard 7-day triple therapy in Taiwan $[9,10]$. In the World Gastroenterology Organization Global Guidelines, bismuth-containing quadruple therapy including PPI-bismuth plus two antibiotics, either tetracycline and metronidazole or amoxicillin and clarithromycin, is recommended as one of the first-line treatments in developing countries [11]. A study in Thailand showed $92 \%$ eradication rate via adding bismuth to the standard 7-day triple therapy [12]. However, a poor eradication efficacy $(\sim 68 \%)$ using such an approach was reported in China [13]. Since the clarithromycin resistance is still less than $15 \%$ in Taiwan, we aimed to investigate whether adding bismuth to 7-day rabeprazole-based standard triple therapy can increase the eradication efficacy.

\section{Patients and Methods}

2.1. Patients. From April 2013 to January 2015, patients who visited the Gastroenterology Clinic of Ten Chan Hospital with complaints of dyspepsia or epigastric discomfort were invited to participate in this study. All of them were aged over 20 years and received upper gastrointestinal endoscopy to confirm the diagnosis and positive $H$. pylori infection. Those who had taken antibiotics, bismuth, or proton pump inhibitors within the previous 4 weeks were excluded. Other exclusion criteria included allergy to any drug in the study, prior gastric surgery, severe concomitant diseases (e.g., decompensate liver cirrhosis, uremia, and gastric cancer), previous $H$. pylori eradication therapy, pregnancy, or lactation.

2.2. Study Design. The study flow chart is shown in Figure 1. All participants underwent upper endoscopic examinations; biopsy samples were taken from gastric body and antrum to detect $H$. pylori by rapid urease test, histology, and culture. The presence of $H$. pylori was defined as (i) a positive result of culture or (ii) positive results of both rapid urease test and histology. The participants were interviewed by trained study nurses using a standardized questionnaire to obtain their demographic data and medical history.

Once confirmed with $H$. pylori infection and having signed the informed consent, participants were randomly assigned to either the standard triple-therapy group (RAK: rabeprazole $20 \mathrm{mg}$, amoxicillin $1 \mathrm{~g}$, and clarithromycin $500 \mathrm{mg}$, all twice daily for 7 days) or the bismuth plus standard triple-therapy group (RBAK: rabeprazole $20 \mathrm{mg}$, bismuth subcitrate $360 \mathrm{mg}$, amoxicillin $1 \mathrm{~g}$, and clarithromycin $500 \mathrm{mg}$, all twice daily for 7 days). A computergenerated random number was chosen for randomization. The patients and physicians were not blinded to the therapy assigned.

After completion of $H$. pylori eradication therapy, participants were asked to come back for collection of the information on any adverse event of drug compliance. In order to avoid false negative results, they were also asked to have a 4-week PPI, antibiotic, and bismuth washout period before further examination of $H$. pylori status. The second endoscopy with rapid urea test, histology, and culture or ${ }^{13} \mathrm{C}$-urea breath test (UBT) for those who refused endoscopic exams was carried out at the end of the washout period. Those who did not return to confirm their H. pylori status were deemed as treatment failure in intention-to-treat (ITT) analysis. This study was approved by the Institutional Review Board of Kaohsiung Medical University Hospital (KMUH-IRB-20120028), Kaohsiung, Taiwan. This study has been registered to the ClinicalTrials.gov Protocol Registration and Result System (PRS); the registry number was NCT03108287. 
2.3. Questionnaire. The questionnaire used here covered the participants' personal and medical history, adverse effects, and compliances. Smokers were defined as those who smoked more than one pack of cigarettes per week. Alcohol drinkers were defined as those who had at least one cup of alcoholic beverage 3 days a week. The adverse effects included abdominal pain, diarrhea, constipation, headache, poor appetite, nausea/vomiting, skin rash, dizziness, taste change, and others. The participants who deemed these newly onset symptoms disturbing were recognized as having major adverse effects, and those who did not consider the symptoms as constituting a disturbance to their daily life were defined as having minor adverse effects. Compliance was evaluated by counting the remaining medication at the end of treatment. Poor compliance was defined as consumption of less than $70 \%$ of the prescription medication.

\subsection{Diagnosis of H. pylori Infection}

2.4.1. Pathological Examination and Culture. Biopsy specimens taken from the lesser curvature of the gastric antrum and angularis were sent for pathology with hematoxylin and eosin stain and bacterial cultures. The pathologists read the results and diagnosed the severity of gastritis according to the updated Sydney classification. For culture, the tissues were rubbed on a Columbia blood agar plate and incubated at $35^{\circ} \mathrm{C}$ under microaerobic conditions for 4-5 days. A positive culture of $H$. pylori was considered in the presence of Gram-negative, oxidase, catalase, and urease-positive colonies.

2.4.2. Rapid Urease Test. The detailed method has been described previously [14]. In brief, the Campylobacter-like organism test (Delta West, Perth, WA, Australia) was used and the results were obtained 24 hours after examination.

2.4.3. ${ }^{13} \mathrm{C}$-Urea Breath Test (UBT). The ${ }^{13} \mathrm{C}$-urea was manufactured by the Institute of Nuclear Energy Research in Taiwan, and the protocol was described in the previous report [14]. One hundred $\mathrm{mg}$ of $99 \%{ }^{13} \mathrm{C}$-labeled urea was dissolved in $50 \mathrm{ml}$ sterile water. The expired air was collected before and $30 \mathrm{~min}$ after the patients drank the water containing ${ }^{13} \mathrm{C}$-labeled urea, and the samples were analyzed. The cut-off value for a positive result was an increase of 4 per mil of ${ }^{13} \mathrm{CO}_{2}$ in the $30 \mathrm{~min}$ sample subtracting that in the baseline sample. The technician who performed UBT and read the results was blinded to the regimen assigned.

2.4.4. Antimicrobial Resistance. After H. pylori was successfully cultured, it was sent for examination of antibiotic resistance. The detailed methods and criteria chosen as the minimal inhibitory concentration were based on the previous report [15]. A Campy-blood agar plate [Brucella agar (Difco, Detroit, MI, USA) + Iso-Vitalex (Gibco, Grand Island, NY, USA) $+10 \%$ whole sheep blood] was used to isolate resistance strains of $H$. pylori. Epsilometer test (E-test: AB Biodisck, Solna, Sweden) was used to test antibiotic resistance against clarithromycin, tetracycline, metronidazole, amoxicillin, and levofloxacin. $H$. pylori strains with minimal inhibitory concentrations $>1,>4,>8,>0.5$, and $>1 \mathrm{mg} / \mathrm{L}$ were
TABLE 1: Characteristics of RAK and RBAK therapies.

\begin{tabular}{lccc}
\hline & RAK & RBAK & $p$ value \\
\hline Patients $(N)$ & 81 & 81 & \\
Male/female $(n)$ & $35 / 46$ & $40 / 41$ & 0.43 \\
Age (years, mean \pm SD) & $49.80 \pm 14.4$ & $51.16 \pm 12.5$ & 0.26 \\
NSAIDs, $n / N(\%)$ & $6 / 81(7.4)$ & $6 / 81(7.4)$ & - \\
Prednisolone use, $n / N(\%)$ & $1 / 81(1.2)$ & $1 / 81(1.2)$ & - \\
Anticoagulant, $n / N(\%)$ & $0 / 81(0)$ & $0 / 81(0)$ & - \\
Smoke, $n / N(\%)$ & $23 / 81(28.4)$ & $20 / 81(24.7)$ & 0.60 \\
Alcohol, $n / N(\%)$ & $10 / 81(12.3)$ & $6 / 81(7.4)$ & 0.29 \\
Gastritis, $n / N(\%)$ & $68 / 81(84.0)$ & $72 / 81(88.9)$ & 0.36 \\
GU, $n / N(\%)$ & $16 / 81(19.8)$ & $17 / 81(21.0)$ & 0.84 \\
DU, $n / N(\%)$ & $32 / 81(39.5)$ & $35 / 81(43.2)$ & 0.63 \\
GU $+\mathrm{DU}, n / N(\%)$ & $4 / 81(4.9)$ & $12 / 81(14.8)$ & 0.04 \\
\hline
\end{tabular}

considered to be resistant to clarithromycin, tetracycline, metronidazole, amoxicillin, and levofloxacin, respectively.

2.4.5. Statistical Analysis. All the data was analyzed using Stat View 5.0.1 (SAS Institute, Cary, NC, USA). A $\chi^{2}$ test and Student's $t$-test were used in this study. It was evaluated by ITT and per-protocol (PP) analysis as the major outcome for the rate of successful $H$. pylori eradication. Independent factors were evaluated by univariate analysis and confirmed by logistic regression. A $p$ value $<0.05$ was considered statistically significant. Assuming that the conventional eradication rate (RAK group) is 77\% [9], and the RBAK group was to achieve $85 \%$ eradication rate, an $8 \%$ difference of increase, our statistical power would be $85 \%$ under the sample sizes of about 198 subjects in each group and two-sided $p$ value of 0.05 if $95 \%$ of patients $(N=188)$ completed the follow-up [16].

\section{Results}

This study was terminated early because the interim analysis showed a lower efficacy of the RBAK regimen ( $78 \%$ in ITT analysis) than we expected. In total, $178 \mathrm{H}$. pylori-infected patients were enrolled and 16 were excluded due to pregnancy or refusal to participate (Figure 1). Eighty-one of the remaining 162 participants were randomized into the RAK group, while the other half was placed in the RBAK group. There were no significant differences in age, gender, smoking and alcohol consumption, anticoagulant, nonsteroidal antiinflammatory drugs (NSAIDs), and prednisolone use in both groups (Table 1). In ITT analysis, the eradication rates was $72.8 \%(59 / 81)$ in the RAK group and $77.8 \%(63 / 81)$ in the RBAK group $(p=0.47$, Table 2$)$. PP analysis was $74.7 \%$ $(59 / 79)$ in the RAK group and $81.8 \%(63 / 77)$ in the RBAK group $(p=0.28)$. Six patients took less than $70 \%$ of the total medication and were deemed to have poor compliance. The compliance was $97.5 \%$ in the RAK group and $95.1 \%$ in the RBAK group ( $p=0.40$, Table 2). Similar prevalence of overall (both 50.6\%, Table 2) and detailed side effects (Table 3) was observed. The most common side effects were bad taste, followed by diarrhea, and abdominal pain. All of the events subsided spontaneously or were medically manageable. 
TABLE 2: Major outcomes of RAK and BRAK for Helicobacter pylori eradication.

\begin{tabular}{lccc}
\hline \multicolumn{2}{c}{ RAK } & BRAK & $p$ value \\
\hline Eradication rate, $n / N(\%)$ & & & \\
$\quad$ Intention-to-treat & $59 / 81(72.8)$ & $63 / 81(77.8)$ & 0.47 \\
$\quad$ Per protocol & $59 / 79(74.7)$ & $63 / 77(81.8)$ & 0.28 \\
Compliance, $n / N(\%)$ & $79 / 81(97.5)$ & $77 / 81(95.1)$ & 0.40 \\
Side effect, $n / N(\%)$ & $41 / 81(50.6)$ & $41 / 81(50.6)$ & - \\
\hline
\end{tabular}

7 patients had an unknown $H$. pylori status after treatment (3 in RAK group, 4 in RBAK group).

TABLE 3: Adverse events of RAK and RBAK therapies for Helicobacter pylori eradication.

\begin{tabular}{lccc}
\hline Adverse events, $n(\%)$ & RAK $(N=81)$ & RBAK $(N=81)$ & $p$ value \\
\hline Abdominal pain & $9(11.1)$ & $12(14.8)$ & 0.48 \\
Diarrhea & $14(17.3)$ & $11(13.6)$ & 0.52 \\
Constipation & $3(3.7)$ & $4(4.9)$ & 0.70 \\
Headache & $3(3.7)$ & $1(1.2)$ & 0.31 \\
Anorexia & $1(1.2)$ & $1(1.2)$ & - \\
Nausea & $3(3.7)$ & $2(2.5)$ & 0.65 \\
Skin rash & $2(2.5)$ & $1(1.2)$ & 0.56 \\
Dizziness & $3(3.7)$ & $2(2.5)$ & 0.65 \\
Bad taste & $25(30.9)$ & $20(24.7)$ & 0.38 \\
Fatigue & $9(11.1)$ & $7(8.6)$ & 0.60 \\
Others & $7(8.6)$ & $15(18.5)$ & 0.07 \\
Overall & $41(50.6)$ & $41(50.6)$ & - \\
\hline
\end{tabular}

H. pylori strains were successfully isolated from $50 \%$ (81/ 162) of the participants (Table 4). The overall antimicrobial resistance rates were $27.2 \%$ for metronidazole, $12.3 \%$ for clarithromycin, $0 \%$ for tetracycline, $11.1 \%$ for levofloxacin, and $2.5 \%$ for amoxicillin (Table 4 ). The prevalence of antibiotic resistance was similar in both groups. Clinical factors, including antibiotic resistance, presence of dual resistance, adverse effects, compliance, and smoking, that might influence the eradication rate of the two regimens were examined (Table 5). Although not statistically significant, presence of clarithromycin resistance did result in a very low eradication efficacy in both groups ( $40 \%$ versus $20 \%, p=1.00)$. Dual resistance, defined as resistance to any two of the five antibiotics in the table, was also related to a lower success rate in $H$. pylori treatment (ITT: 60\% in both groups, Table 5).

\section{Discussion}

Our results showed slightly higher but not satisfactory eradication results by adding bismuth to the standard 7-day rabeprazole-based triple therapy in Northern Taiwan. A recent study in Taiwan reported an $83.7 \%$ eradication rate using PPI-amoxicillin-clarithromycin for 14 days [17]. The bismuth quadruple therapy (bismuth dicitrate $300 \mathrm{mg}$ qid, lansoprazole $30 \mathrm{mg}$ bid, tetracycline $500 \mathrm{mg}$ qid, and metronidazole $500 \mathrm{mg}$ tid for 10 days) appeared to be a better
TABLE 4: Rate of antimicrobial resistance of Helicobacter pylori.

\begin{tabular}{lcccc}
\hline $\begin{array}{l}\text { Antimicrobial } \\
\text { resistance, } n(\%)\end{array}$ & $\begin{array}{c}\text { RAK } \\
(N=38)\end{array}$ & $\begin{array}{c}\text { BRAK } \\
(N=43)\end{array}$ & $p$ value & $\begin{array}{c}\text { Total } \\
(N=81)\end{array}$ \\
\hline Etronidazole & $11(28.9)$ & $11(25.6)$ & 0.73 & $22(27.2)$ \\
Clarithromycin & $5(13.2)$ & $5(11.6)$ & 0.83 & $10(12.3)$ \\
Tetracycline & $0(0.0)$ & $0(0.0)$ & - & $0(0.0)$ \\
Levofloxacin & $3(7.9)$ & $6(14.0)$ & 0.38 & $9(11.1)$ \\
Amoxicillin & $1(2.6)$ & $1(2.3)$ & 0.93 & $2(2.5)$ \\
\hline
\end{tabular}

TABLE 5: Univariate analysis of clinical factors which might influence the efficacy of the RAK and RBAK.

\begin{tabular}{|c|c|c|c|}
\hline Eradication rate, $n / N(\%)$ & RAK & RBAK & $p$ value \\
\hline \multicolumn{4}{|l|}{ Resistance to } \\
\hline \multicolumn{4}{|l|}{ Metronidazole* } \\
\hline ITT and PP & $6 / 11(54.5)$ & $7 / 11(63.6)$ & 1.00 \\
\hline \multicolumn{4}{|l|}{ Clarithromycin* } \\
\hline ITT and PP & $2 / 5(40.0)$ & $1 / 5(20.0)$ & 1.00 \\
\hline Tetracycline & - & - & - \\
\hline Levofloxacin* & $3 / 3(100)$ & $3 / 6(50.0)$ & 0.46 \\
\hline Amoxicillin* & $1 / 1(100)$ & $1 / 1(100)$ & 1.00 \\
\hline \multicolumn{4}{|l|}{ Dual resistance } \\
\hline \multicolumn{4}{|l|}{ Present* } \\
\hline ITT & $3 / 5(60.0)$ & $3 / 5(60.0)$ & 1.00 \\
\hline $\mathrm{PP}$ & $3 / 5(60.0)$ & $3 / 4(75.0)$ & 1.00 \\
\hline \multicolumn{4}{|l|}{ Absent } \\
\hline ITT & $27 / 33(81.8)$ & $27 / 38(71.1)$ & 0.29 \\
\hline $\mathrm{PP}$ & $27 / 31(87.1)$ & $27 / 35(77.1)$ & 0.30 \\
\hline \multicolumn{4}{|l|}{ Adverse events } \\
\hline Present & $25 / 36(69.4)$ & $34 / 41(82.9)$ & 0.16 \\
\hline Absent & $31 / 40(77.5)$ & $29 / 40(72.5)$ & 0.61 \\
\hline \multicolumn{4}{|l|}{ Compliance } \\
\hline Good & $58 / 80(72.5)$ & $63 / 81(77.8)$ & 0.44 \\
\hline Poor* & $1 / 1(100)$ & $0 / 0(0.0)$ & - \\
\hline \multicolumn{4}{|l|}{ Smoking } \\
\hline Present & $17 / 23(73.9)$ & $15 / 20(75.0)$ & 0.94 \\
\hline Absent & $42 / 58(72.4)$ & $48 / 61(78.7)$ & 0.42 \\
\hline
\end{tabular}

${ }^{*}$ Fisher exact test. ${ }^{9}$ Dual resistance was defined as presence of any two of the five antibiotics.

first-line treatment (90.4\% by ITT analysis) [17]. However, their clarithromycin resistance rate was higher (14-17\%) than our study $(12.3 \%)$. In Thailand, there was a high eradication rate (92-100\%) using 7- or 14-day triple therapy (lansoprazole, amoxicillin, and clarithromycin) plus bismuth with or without probiotics [12]. However, the sample size was small (25 cases in each group) and their clarithromycin resistance rate was very low $(<4 \%)$. Another study in mainland China did not find adding bismuth to the 10-day triple therapy an effective regimen $(68.4 \%$ versus $68 \%$ by ITT analysis) [18]. Different antibiotic resistance rates across areas could be an important cause for such discrepancies. 
According to Maastricht $\mathrm{V}$ Consensus, bismuthcontaining quadruple treatments are the recommended first-line therapy in areas of high clarithromycin resistance [5]. It was also suggested that PPI-clarithromycincontaining triple therapy should be abandoned in areas with clarithromycin resistance rate over 15\% (low evidence level, strongly recommended) [5]. The clarithromycin resistance rate was $12.3 \%$ in the present study. However, the eradication rate was only $72.8 \%$ by ITT analysis for 7 -day triple therapy; adding bismuth increased the success rate by $5 \%$. Further extending the duration of RBAK regimen to 10-14 days or substitute clarithromycin with another antibiotic may be a better strategy. In a meta-analysis, 10-day bismuth quadruple therapy had better efficacy than 7-day standard triple therapy while there were similar compliance and side effect rates [19]. Ciccaglione et al. indicated that adding bismuth to pantoprazole, amoxicillin and moxifloxacin had achieved a significantly higher eradication rate $(92 \%$ versus $77.1 \%, p<0.03)$ [20]. In another study, the moxifloxacin-tetracycline-lansoprazole regimen plus bismuth was a better regimen than that without bismuth (82.1\% versus 55.4\%) [21]. Because bismuth citrates inhibit the motility of $H$. pylori, cause morphological destruction, and suppress the growth of $H$. pylori, it can be a component in $H$. pylori eradication regimen [22].

With the increasing antibiotic usage, drug resistance has become a global issue and substantially influences the efficacy of $H$. pylori eradication therapy [23]. In Europe, the drug resistance rate was $17.5 \%$ for clarithromycin, $34.9 \%$ for metronidazole, and the rate of clarithromycin resistance was different across European countries [24]. Resistance to clarithromycin, metronidazole, and fluoroquinolone is also increasing in China [13]. In Taiwan, the clarithromycin resistance rate in our study was very similar to some reports $(11.4-12.8 \%)[8,25]$, but lower than that $(14-17 \%)$ in a recent large-scale study [17]. This suggested that local clarithromycin resistance, even in the same country, is a key factor when choosing the most appropriate first-line treatment for $H$. pylori.

In conclusion, with the increasing clarithromycin resistance in Taiwan, 7-day triple therapy may no longer be a suitable first-line therapy even after adding bismuth. Further study is needed to clarify whether extending the duration of RBAK regimen to 10-14 days can lead to a better result.

\section{Conflicts of Interest}

The authors declare that they have no conflicts of interest.

\section{Authors' Contributions}

Yao-Kang Huang and I-Chen Wu contributed equally to this work.

\section{Acknowledgments}

This study was supported by grants from the Ten Chan General Hospital, Chung-Li and KMU Joint Research Project (ST102004), Kaohsiung Medical University Hospital
(KMUH99-9M68, 10501), and Kaohsiung Municipal TaTung Hospital (kmtth-104-008).

\section{References}

[1] NIH Consensus Conference, "Helicobacter pylori in peptic ulcer disease. NIH Consensus development panel on Helicobacter pylori in peptic ulcer disease," Jama, vol. 272, no. 1, pp. 65-69, 1994.

[2] European Helicobacter Pylori Study Group, "Current European concepts in the management of Helicobacter pylori infection. The Maastricht Consensus report," Gut, vol. 41, no. 1, pp. 8-13, 1997.

[3] E. Rimbara, L. A. Fischbach, and D. Y. Graham, "Optimal therapy for Helicobacter pylori infections," Nature Reviews Gastroenterology \& Hepatology, vol. 8, no. 2, pp. 79-88, 2011.

[4] D. Y. Graham and L. Fischbach, "Helicobacter pylori treatment in the era of increasing antibiotic resistance," Gut, vol. 59, no. 8, pp. 1143-1153, 2010.

[5] P. Malfertheiner, F. Megraud, C. A. O'Morain et al., "Management of Helicobacter pylori infection-the Maastricht V/Florence Consensus report," Gut, vol. 66, no. 1, pp. 6-30, 2017.

[6] S. L. Gorbach, "Bismuth therapy in gastrointestinal diseases," Gastroenterology, vol. 99, no. 3, pp. 863-875, 1990.

[7] I. Thung, H. Aramin, V. Vavinskaya et al., "Review article: the global emergence of Helicobacter pylori antibiotic resistance," Alimentary Pharmacology \& Therapeutics, vol. 43, no. 4, pp. 514-533, 2016.

[8] J. M. Liou, C. Y. Chang, M. J. Chen et al., "The primary resistance of Helicobacter pylori in Taiwan after the National Policy to restrict antibiotic consumption and its relation to virulence factors-a nationwide study," PloS One, vol. 10, no. 5, article e0124199, 2015.

[9] C. C. Chan, N. H. Chien, C. L. Lee et al., "Comparison of 10day sequential therapy with 7-day standard triple therapy for Helicobacter pylori eradication in inactive peptic ulcer disease and the efficiency of sequential therapy in inactive peptic ulcer disease and non-ulcer dyspepsia," BMC Gastroenterology, vol. 15, p. 170, 2015.

[10] P. I. Hsu, D. C. Wu, W. C. Chen et al., "Randomized controlled trial comparing 7-day triple, 10-day sequential, and 7-day concomitant therapies for Helicobacter pylori infection," Antimicrobial Agents and Chemotherapy, vol. 58, no. 10, pp. 5936-5942, 2014.

[11] R. H. Hunt, S. D. Xiao, F. Megraud et al., "Helicobacter pylori in developing countries. World gastroenterology organisation global guideline," Journal of Gastrointestinal and Liver Diseases, vol. 20, no. 3, pp. 299-304, 2011.

[12] C. Srinarong, S. Siramolpiwat, A. Wongcha-um, V. Mahachai, and R. K. Vilaichone, "Improved eradication rate of standard triple therapy by adding bismuth and probiotic supplement for Helicobacter pylori treatment in Thailand," Asian Pacific Journal of Cancer Prevention, vol. 15, no. 22, pp. 9909-9913, 2014.

[13] W. Gao, H. Cheng, F. Hu et al., "The evolution of Helicobacter pylori antibiotics resistance over 10 years in Beijing, China," Helicobacter, vol. 15, no. 5, pp. 460-466, 2010.

[14] I. C. Wu, H. L. Ke, Y. C. Lo et al., "Evaluation of a newly developed office-based stool test for detecting Helicobacter pylori: an extensive pilot study," Hepato-Gastroenterology, vol. 50, no. 54, pp. 1761-1765, 2003. 
[15] T. Alarcon, D. Domingo, and M. Lopez-Brea, "Antibiotic resistance problems with Helicobacter pylori," International Journal of Antimicrobial Agents, vol. 12, no. 1, pp. 19-26, 1999.

[16] D. Machin, M. Campbell, P. Fayers, and A. Pinol, Sample Size Tables for Clinical Studies, 2nd Edition, Blackwell Science, UK, 1997.

[17] J. M. Liou, Y. J. Fang, C. C. Chen et al., "Concomitant, bismuth quadruple, and 14-day triple therapy in the first-line treatment of Helicobacter pylori: a multicentre, open-label, randomised trial," Lancet, vol. 388, no. 10058, pp. 2355-2365, 2016.

[18] J. Liang, J. Li, Y. Han et al., "Helicobacter pylori eradication with ecabet sodium, omeprazole, amoxicillin, and clarithromycin versus bismuth, omeprazole, amoxicillin, and clarithromycin quadruple therapy: a randomized, open-label, phase IV trial," Helicobacter, vol. 17, no. 6, pp. 458-465, 2012.

[19] M. Venerito, T. Krieger, T. Ecker, G. Leandro, and P. Malfertheiner, "Meta-analysis of bismuth quadruple therapy versus clarithromycin triple therapy for empiric primary treatment of Helicobacter pylori infection," Digestion, vol. 88, no. 1, pp. 33-45, 2013.

[20] A. F. Ciccaglione, L. Cellini, L. Grossi, and L. Marzio, "Quadruple therapy with moxifloxacin and bismuth for first-line treatment of Helicobacter pylori," World Journal of Gastroenterology, vol. 18, no. 32, pp. 4386-4390, 2012.

[21] B. Ergul, E. Kocak, A. Tas, L. Filik, and S. Koklu, "Bismuth, moxifloxacin, tetracycline, lansoprazole quadruple first line therapy for eradication of H. pylori: a prospective study," Clinics and Research in Hepatology and Gastroenterology, vol. 37, no. 5, pp. 527-529, 2013.

[22] M. L. Worku, R. L. Sidebotham, and Q. N. Karim, "Effects of ranitidine bismuth citrate on Helicobacter pylori motility, morphology and survival," Alimentary Pharmacology \& Therapeutics, vol. 13, no. 6, pp. 753-760, 1999.

[23] L. Fischbach and E. L. Evans, "Meta-analysis: the effect of antibiotic resistance status on the efficacy of triple and quadruple first-line therapies for Helicobacter pylori," Alimentary Pharmacology \& Therapeutics, vol. 26, no. 3, pp. 343-357, 2007.

[24] F. Megraud, S. Coenen, A. Versporten et al., "Helicobacter pylori resistance to antibiotics in Europe and its relationship to antibiotic consumption," Gut, vol. 62, no. 1, pp. 34-42, 2013.

[25] Y. K. Huang, M. C. Wu, S. S. Wang et al., "Lansoprazole-based sequential and concomitant therapy for the first-line Helicobacter pylori eradication," Journal of Digestive Diseases, vol. 13, no. 4, pp. 232-238, 2012. 


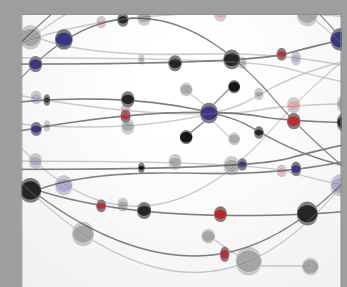

The Scientific World Journal
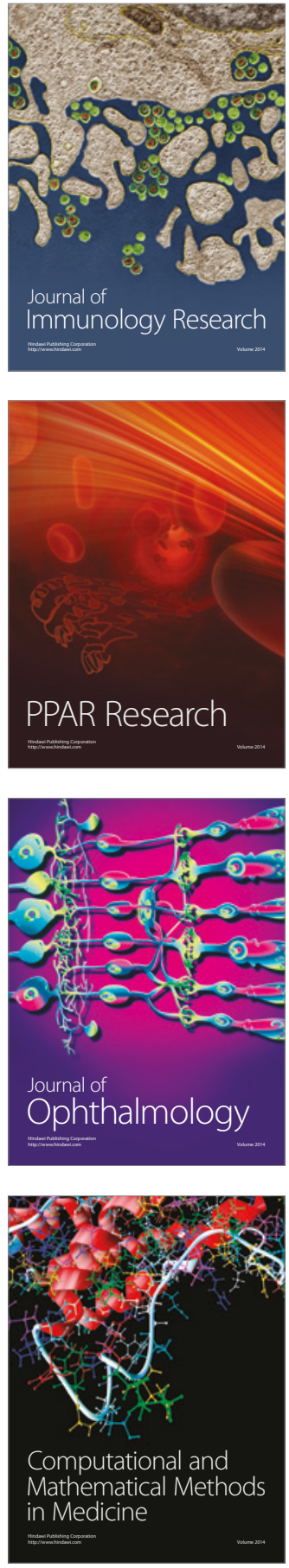

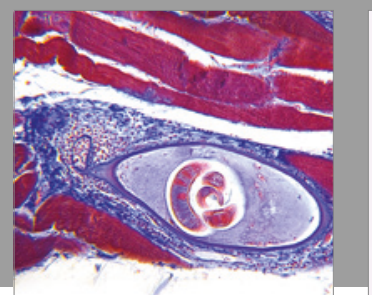

Gastroenterology Research and Practice
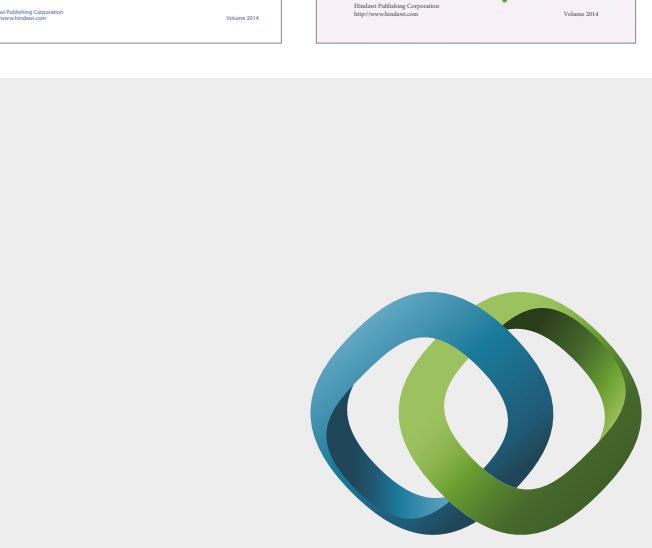

\section{Hindawi}

Submit your manuscripts at

https://www.hindawi.com
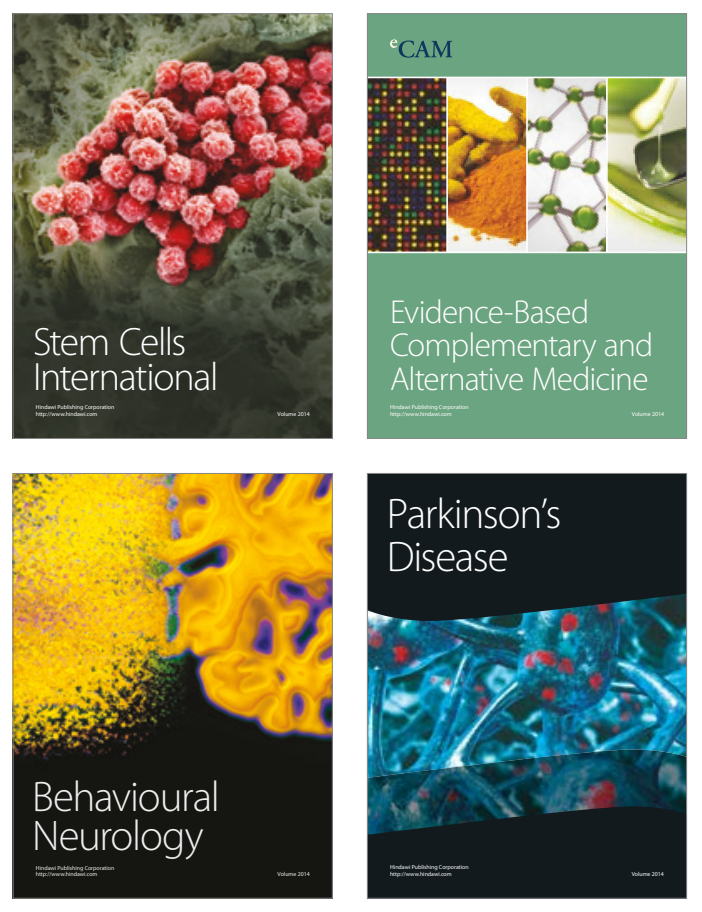
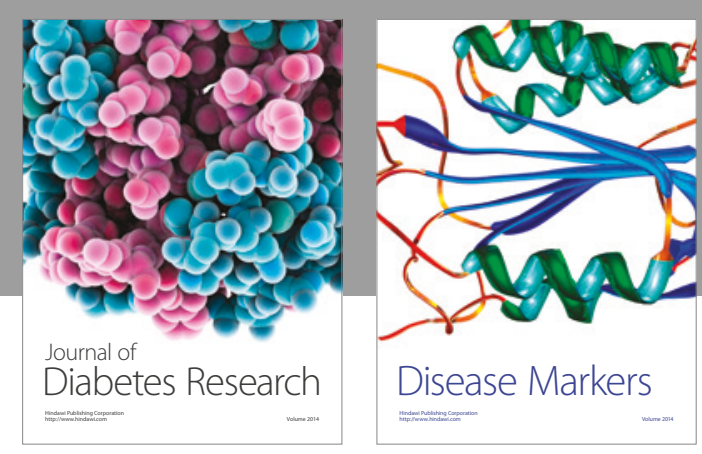

Disease Markers
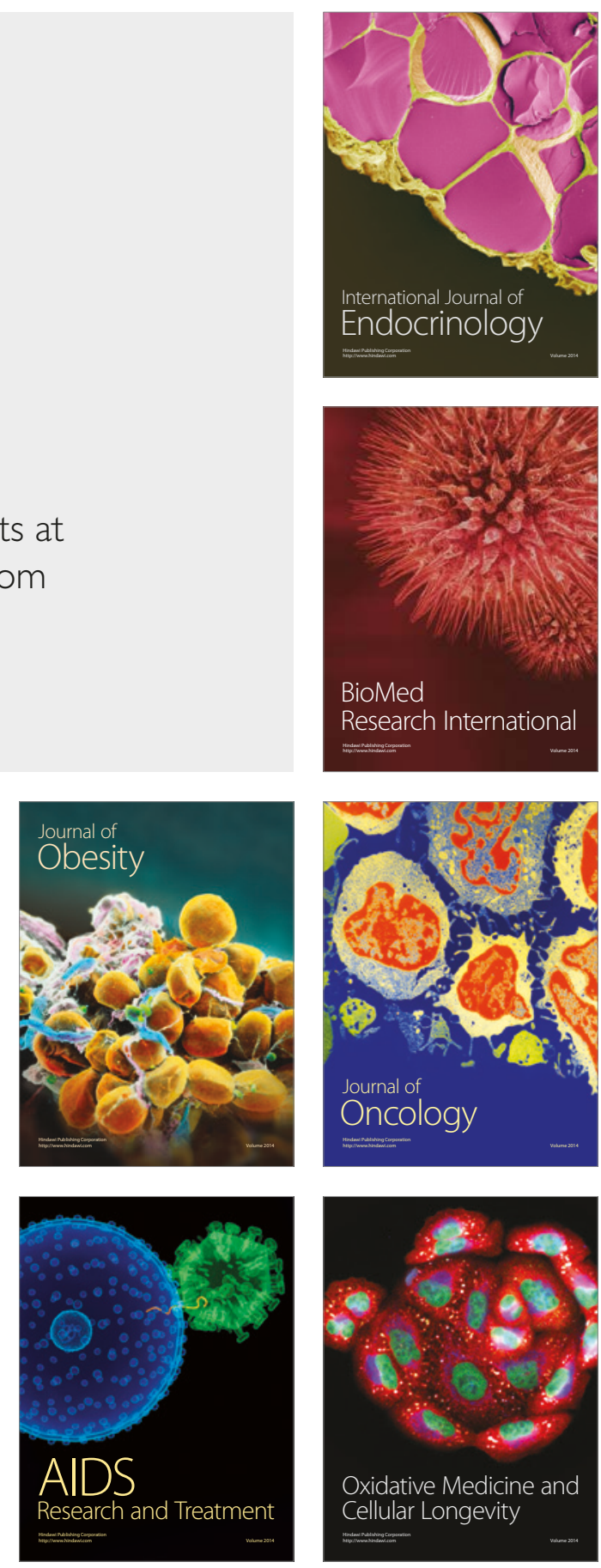